\title{
Role of NKG2D in Obesity-Induced Adipose Tissue Inflammation and Insulin Resistance
}

\section{Jun-Jae Chung ${ }^{1}$, Mary A. Markiewicz ${ }^{1 \times}$, Bojan Polić ${ }^{2}$, Andrey S. Shaw ${ }^{1,3 *}$}

1 Department of Pathology and Immunology, Washington University School of Medicine, St. Louis, Missouri, United States of America, 2 Department of Histology and Embryology, University of Rijeka School of Medicine, Rijeka, Croatia, 3 Howard Hughes Medical Institute, Washington University School of Medicine, St. Louis, Missouri, United States of America

\begin{abstract}
The early events that initiate inflammation in the adipose tissue during obesity are not well defined. It is unclear whether the recruitment of CD8 T cells to the adipose tissue during onset of obesity occurs through antigen-dependent or independent processes. We have previously shown that interaction between NKG2D (natural-killer group 2, member D) and its ligand Rae-1 $\varepsilon$ is sufficient to recruit cytotoxic $T$ lymphocytes to the pancreas and induce insulitis. Here, we tested whether NKG2D-NKG2D ligand interaction is also involved in obesity-induced adipose tissue inflammation and insulin resistance. We observed a significant induction of NKG2D ligand expression in the adipose tissue of obese mice, especially during the early stages of obesity. However, mice lacking NKG2D developed similar levels of insulin resistance and adipose tissue inflammation compared to control mice when placed on a high-fat diet. Moreover, overexpression of Rae- $1 \varepsilon$ in the adipose tissue did not increase immune cell infiltration to the adipose tissue either in the setting of a normal or high-fat diet. These results indicate that, unlike in the pancreas, NKG2D-NKG2D ligand interaction does not play a critical role in obesity-induced inflammation in the adipose tissue.
\end{abstract}

Citation: Chung J-J, Markiewicz MA, Polić B, Shaw AS (2014) Role of NKG2D in Obesity-Induced Adipose Tissue Inflammation and Insulin Resistance. PLoS ONE 9(10): e110108. doi:10.1371/journal.pone.0110108

Editor: Ayyalasomayajula Vajreswari, National Institute of Nutrition, India

Received April 23, 2014; Accepted September 16, 2014; Published October 15, 2014

Copyright: (C) 2014 Chung et al. This is an open-access article distributed under the terms of the Creative Commons Attribution License, which permits unrestricted use, distribution, and reproduction in any medium, provided the original author and source are credited.

Data Availability: The authors confirm that all data underlying the findings are fully available without restriction. All relevant data are within the paper.

Funding: This research was supported by the Howard Hughes Medical Institute and National Institutes of Health grant R01DK058366. The funders had no role in study design, data collection and analysis, decision to publish, or preparation of the manuscript.

Competing Interests: The authors have declared that no competing interests exist.

*Email: shaw@pathology.wustl.edu

a Current address: Department of Microbiology, Molecular Genetics and Immunology, University of Kansas School of Medicine, Kansas City, Kansas, United States of America

\section{Introduction}

Recent studies have pointed to chronic inflammation in insulin target tissues, such as muscle, liver, and adipose tissue, as one of the causal links between obesity and insulin resistance [1-3]. Multiple inflammatory cytokines (TNF- $\alpha$, IL-6, etc.) and signaling pathways (JNK, NF- $\mathrm{BB}$ ) have been implicated in obesity-induced insulin resistance [4-10].

The adipose tissue plays a key role in regulating systemic metabolism. In addition to being a storage depot for lipids, the adipose tissue secretes a number of paracrine and endocrine factors, known as adipocytokines, that modulate metabolism and inflammation in liver, muscle, and pancreatic islets [11]. In obese mice, the secretion profile of the adipose tissue is altered by the pro-inflammatory milieu. Secretion of pro-inflammatory adipocytokines, such as resistin, IL-6, and TNF- $\alpha$ is increased while antiinflammatory and insulin-sensitizing adipocytokines like adiponectin are down-regulated. It is therefore thought that dysregulation of the adipose tissue during obesity induces insulinresistance and inflammation in major metabolic tissues.

Despite much effort, the initiating events that cause inflammation in obese adipose tissue are yet to be clearly understood. Hypoxia and ER stress have been shown to activate inflammatory signaling pathways in obese adipose tissue, but it is unclear whether they are the primary switch that triggers inflammation [12-16].

Various immune cell types have been implicated in obesityinduced insulin resistance [17]. Among them, macrophages are considered to be the major mediators of adipose tissue inflammation $[18,19]$. Macrophages can constitute up to $\sim 40 \%$ of the cell number in the adipose tissue during obesity and are responsible for the majority of Tnfa expression in the adipose tissue [20,21]. Additionally, in contrast to the resident macrophages found in the adipose tissue of lean mice, the macrophages recruited to the adipose tissue during obesity are pro-inflammatory [22]. The resident macrophages display characteristics of "alternatively activated" or M2 macrophages and express anti-inflammatory cytokines such as IL-10, while the newly infiltrating macrophages are of the "classically activated" or M1 category and express high levels of TNF- $\alpha$ and iNOS [22,23].

More recently, $\mathrm{T}$ cells have emerged as a key component in obesity-induced adipose tissue inflammation. Multiple studies have reported increased numbers of CD8 T cells in the adipose tissue of obese rodents and humans along with elevated levels of IFN- $\gamma$ and RANTES, which are important for $\mathrm{T}$ cell function and recruitment [24-29]. Interestingly, accumulation of $T$ cells occurs prior to macrophage infiltration and onset of insulin resistance, and depletion of GD8 T cells alleviates adipose tissue inflamma- 
tion and insulin resistance in obese mice [26,28,30-32]. This suggests that recruitment of pro-inflammatory $\mathrm{T}$ cells could be a primary event that initiates adipose tissue inflammation.

Still, the question of what initiates the infiltration and activation of GD8 T cells in the adipose tissue remains to be answered. It has been suggested that $\mathrm{T}$ cells are responding to chemokines and cytokines produced in the adipose tissue in response to cell death or hypoxia [25,33,34]. A more provocative idea is that certain antigens produced in the adipose tissue under obese conditions could direct immune cell responses. This hypothesis is supported by the observation that adipose tissue $\mathrm{T}$ cells express a restricted repertoire of $\mathrm{T}$ cell receptors (TCRs), whose profile is unique from that of T cells in the spleen or lymph nodes [31-33].

An alternative way of $\mathrm{T}$ cell recruitment and accumulation in tissues is through the interaction between certain high affinity receptors and their ligands independent of antigen. Our lab has previously shown that NKG2D (natural-killer group 2, member $\mathrm{D})$, a cell surface receptor expressed in cytotoxic $\mathrm{T}$ lymphocytes (CTLs), is sufficient to induce recruitment of CTLs to pancreatic islets over-expressing its ligand and cause insulitis [35]. NKG2D binds to a number of distinct ligands which are not expressed in normal adult tissues, but up-regulated in response to stress conditions, such as oxidative stress and viral infection [36]. During obesity, the adipose tissue is subjected to various types of stresses, such as nutrient stress (from elevated glucose and lipid levels), ER stress, oxidative stress, hypoxia, and chronic inflammation $[37,38]$. In this study, we examined whether NKG2D ligand expression is induced in the adipose tissue during obesity. We also tested whether interaction between NKG2D and its cognate ligands plays a role in obesity-induced adipose tissue inflammation and insulin resistance.

\section{Materials and Methods}

\section{Cell Culture}

3T3-L1 fibroblasts (from American Tissue Type Culture Collection) were maintained and differentiated as previously described [39]. For NKG2D ligand expression experiments, differentiated 3T3-L1 cells (day 7, $\sim 95 \%$ differentiated) were treated with $5 \mu \mathrm{g} / \mathrm{ml}$ insulin (Sigma-Aldrich, St. Louis, MO), 50 ng/ml TNF- $\alpha$ (Peprotech, Rocky Hill, NJ), $3 \mu \mathrm{g} / \mathrm{ml}$ tunicamycin (EMD Millipore, Darmstadt, Germany), $50 \mathrm{mU} / \mathrm{ml}$ glucose oxidase (Sigma-Aldrich), 100 ng/ml LPS (Invivogen, San Diego, CA), and $500 \mu \mathrm{M}$ palmitate (Nu-Chek Prep, Elysian, MN) conjugated to $250 \mu \mathrm{M}$ fatty acid-free BSA (SeraCare Life Sciences, Milford, MA) for $24 \mathrm{~h}$.

\section{Mice}

All mice were housed in a specific pathogen-free facility at Washington University School of Medicine. C57BL/6J and $o b / o b$ $\left(\mathrm{B} 6 . \mathrm{V}-\mathrm{Lep} p^{o b} / \mathrm{J}\right)$ mice were purchased from The Jackson Laboratory (Bar Harbor, ME). NKG2D-deficient $\left(K_{\left.l r k 1^{-/}\right)}\right.$mice have been previously described [40]. The PGCALL-Raele mice have been previously described [35]. Adiponectin-Cre transgenic mice were provided by Dr. P. Scherer (University of Texas Southwestern Medical Center) [41]. High-fat diet (HFD) mice were fed the D12492 $60 \mathrm{kcal} \%$ fat diet $(20 \mathrm{kcal} \%$ protein, $20 \mathrm{kcal} \%$ carbohydrate. Each gram contains $5.24 \mathrm{kcal}$, including $228 \mathrm{mg}$ cholesterol from lard) from Research Diets, Inc. (New Brunswick, NJ) for up to 18 weeks. Normal chow control mice were fed a diet of $13 \mathrm{kcal} \%$ fat $(25 \mathrm{kcal} \%$ protein, $62 \mathrm{kcal} \%$ carbohydrate. Each gram contains $3.07 \mathrm{kcal}$ ) from LabDiet (St. Louis, MO). Care and use of mice were conducted in accordance with protocols approved by the Animal Studies Committee at Washington
University in St. Louis (Protocol Number: 20120013), in compliance with the Animal Welfare Act.

\section{Real-time Quantitative PCR Analysis}

RNA was extracted from 3T3-L1 cells, epidydymal fat tissue, or fractionated adipocytes and stromal vascular cells with TRIzol (Life Technologies, Grand Island, NY). RNA was reversetranscribed using random primers with the High Capacity cDNA Reverse Transcription Kit (Life Technologies). Real-time quantitative PCR was performed using the SYBR Green method on the 7500 Fast Real-Time PCR system (Life Technologies). Transcript levels were normalized to HPRT or $\beta$-actin.

The sequence of primers were: Rae-1 pan F - CCA CGT GGG AAT TCA ACA TC; Rae-1 pan R - TGA TGT TGG GTT TTG CTT GG; Mult-1 F - CAA AGG TCT GCT GCT TCA CA; Mult-1 R - TGC TTG TGT CAA CAC GGA AT; H60b F TGG CTG AAG AAA TCG TGA TC; H60b R - CAG TGA GAC CGT GGT TGT CA; CD68 F - TAC CGA ATT CAG GGT GGA AG; CD68 R - ATG GGT ACG GTC ACA ACG TC; TNF- $\alpha$ F - ACG GCA TGG ATC TCA AAG AG, TNF- $\alpha$ R - AGA TAG CAA ATG GGG TGA CG; MCP-1 F - TCG CAA TGA GTA GGC TGG AG; MCP-1 R - TCT GGA CGC ATT CGT TGT TG; adiponectin F - GAC AAG GCG GTT CTC TTG AC; adiponectin R - CAG ACT TGG TCT GCG ACG TG; adipsin F - AGG GAT GGT ATG ATG TGC AG; adipsin R ATT GCA AGG GTA GGG GTC TC; $\beta$-actin F - GAA GAG CTA TGA GCT GCG TGA; $\beta$-actin R - GCA CTG TGT TGG CAT AGA GGT, HPRT F - ATG AGT CAA GGG GGG ACA TA; HPRT R - AGA GGT CGT TTT CAC CAG CA.

\section{Fat Tissue Fractionation}

The epidydymal fat tissue was isolated from euthanized mice, rinsed in PBS, weighed, and minced into small pieces $(\sim 1 \mathrm{~mm})$. The minced tissue was digested in $\mathrm{KRH}$ buffer $(4.8 \mathrm{mM} \mathrm{KCl}$, $2.5 \mathrm{mM} \mathrm{CaCl}_{2}, 1.2 \mathrm{mM} \mathrm{MgSO}_{4}, 118 \mathrm{mM} \mathrm{NaCl}, 20 \mathrm{mM}$ HEPES [pH 7.5]) supplemented with $1.5 \%$ bovine serum albumin (BSA) (SeraCare Life Sciences), $30 \mu \mathrm{g} / \mathrm{ml}$ Liberase (Roche Applied Science, Indianapolis, IN), and $1 \mu \mathrm{g} / \mathrm{ml}$ DNaseI (Sigma-Aldrich) at $37^{\circ} \mathrm{C}$ for $25 \sim 40$ minutes with vigorous shaking. The digested samples were strained through a sterile $250 \mu \mathrm{m}$ nylon mesh (Sefar, Buffalo, NY) and centrifuged at $200 \mathrm{~g}$ for $10 \mathrm{~min}$. The floating cells were collected as the adipocyte fraction and the pelleted cells were collected as the stromal vascular fraction (SVF). The adipocytes were washed twice with $\mathrm{KRH}$ buffer $+1.5 \%$ BSA before being processed for RNA extraction. The SVF was resuspended in ACK lysis buffer $\left(150 \mathrm{mM} \mathrm{NH} \mathrm{NH}_{4}, 10 \mathrm{mM}\right.$ $\mathrm{KHCO}_{3}, 0.1 \mathrm{mM}$ EDTA) and incubated at room temperature for 2 minutes to deplete erythrocytes, washed twice with $\mathrm{KRH}$ buffer $+1.5 \%$ BSA, and finally resuspended in FACS buffer $(1 \%$ BSA, 2 mM EDTA in $1 \times$ PBS) for flow cytometry or processed for RNA extraction.

\section{Isolation of Splenocytes and Peripheral Blood Mononuclear Cells (PBMCs)}

Spleen harvested from euthanized mice were homogenized and filtered through a 40-micron cell strainer. The cells were then washed with PBS, depleted of erythrocytes with ACK lysis buffer, washed twice with PBS, and resuspended in FACS buffer.

For PBMCs, whole blood was collected from mice in EDTAcoated vacutainers (BD Biosciences, San Jose, CA). The blood was mixed with PBS and centrifuged at $300 \mathrm{~g}$ for $10 \mathrm{~min}$. The cells were then resuspended in ACK lysis buffer, washed twice with PBS, and resuspended in FACS buffer. 
A

Rae-1 pan

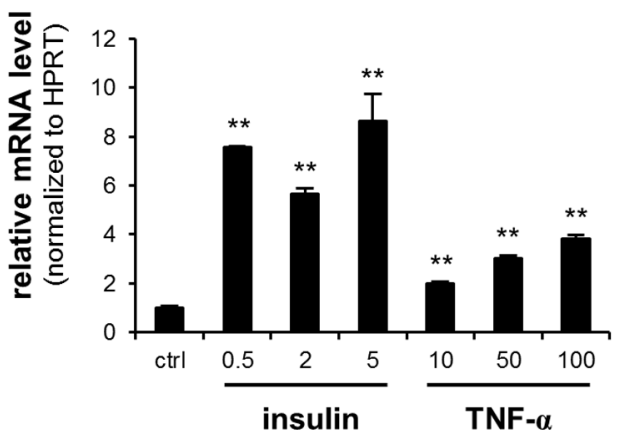

C

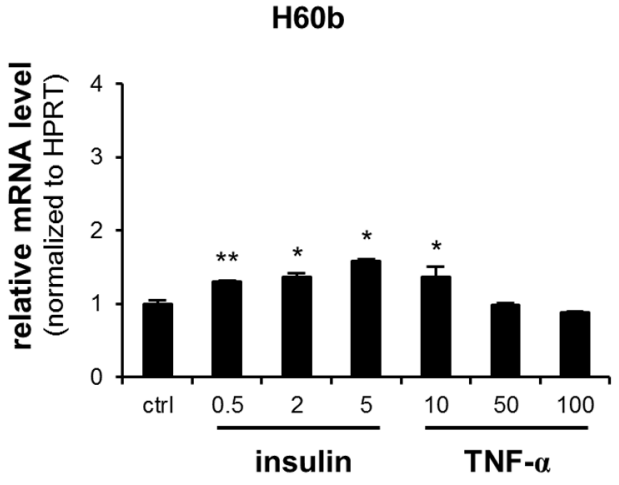

B
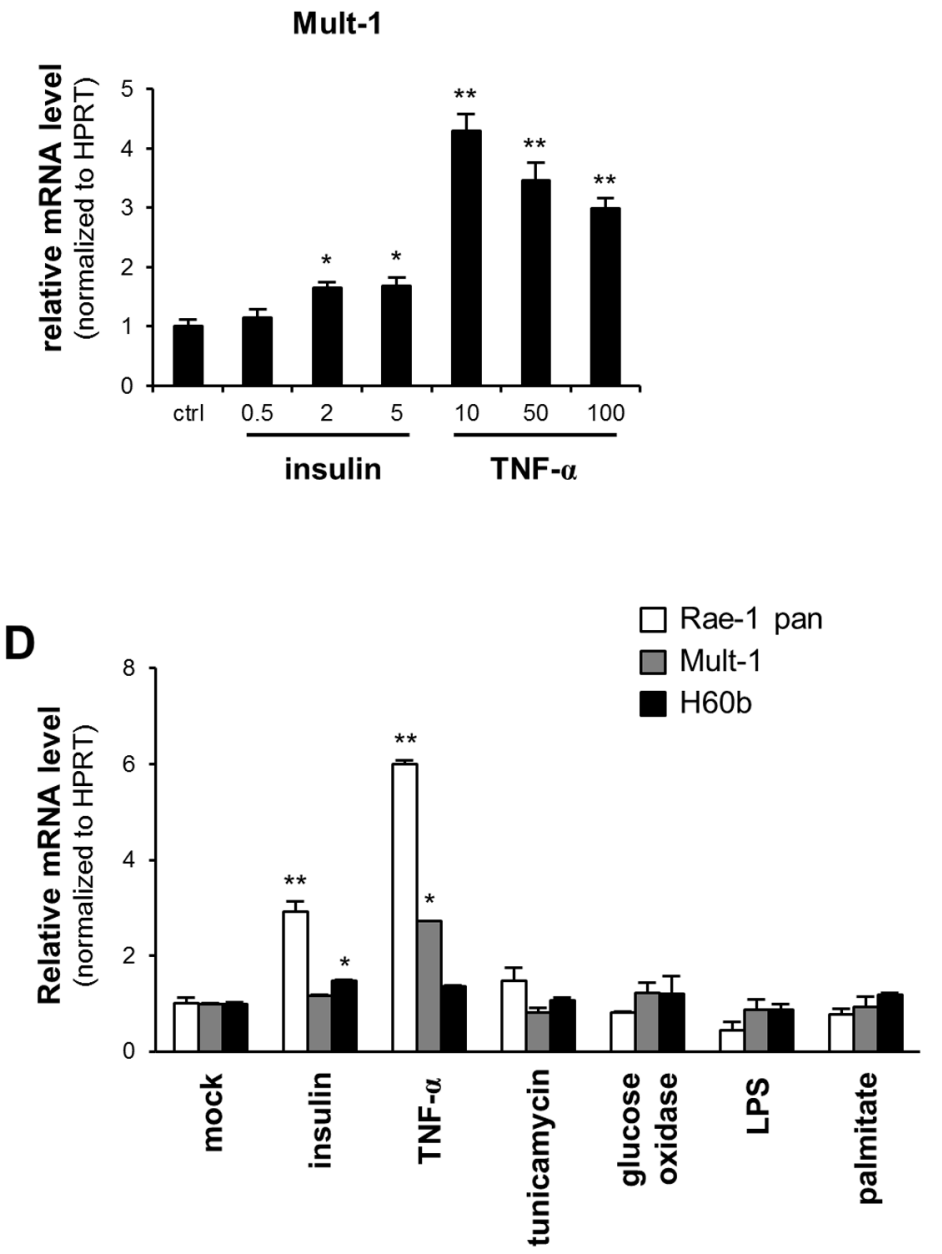

Figure 1. Insulin and TNF- $\alpha$ increases NKG2D ligand expression in 3T3-L1 adipocytes. (A-C) Differentiated 3T3-L1 cells were treated with insulin $(0.5-5 \mu \mathrm{g} / \mathrm{ml})$ or TNF- $\alpha(10-100 \mathrm{ng} / \mathrm{ml})$ for 24 hours. The cells were then analyzed by quantitative PCR to determine the expression levels of (A) Rae-1, (B) Mult-1, and (C) H60b. (D) Differentiated 3T3-L1 cells were treated with insulin, TNF- $\alpha$, tunicamycin, glucose oxidase, LPS, and palmitate. The cells were then analyzed by quantitative PCR for NKG2D ligand expression. The results were normalized to HPRT levels and are shown as foldincrease over control. The data represent mean \pm SD of 3 independent experiments. ${ }^{*} p<0.05,{ }^{* *} p<0.01$ vs. control.

doi:10.1371/journal.pone.0110108.g001

\section{Immunofluorescent Staining and Flow Cytometry}

Single-cell suspensions from the SVF of fat tissue, spleen, and blood samples were incubated in FcBlock (BD Biosciences) for $15 \mathrm{~min}$ at $4^{\circ} \mathrm{C}$. The cells were then stained with fluorophoreconjugated antibodies for $20 \mathrm{~min}$ at $4^{\circ} \mathrm{C}$ in the dark. The antibodies were purchased from BD Biosciences (CD8-FITC, CD11c-FITC, NK1.1-FITC, GD4-PE/Cy7, CD45.2-APG), eBioscience (San Diego, CA) (NKG2D-PE, F4/80-PE/Cy7, CD11b-APG/eFluor780), or BioLegend (San Diego, CA) (CD3e-Pacific Blue). Dead cells were excluded with 7-AAD staining (BD Biosciences). Flow cytometry data was acquired with FACSCantoII or LSRII flow cytometer (BD Biosciences) and analyzed with FlowJo (Treestar, Ashland, OR).

\section{Metabolic Studies}

Male mice were placed on a HFD beginning at 8 weeks of age, and body weight was monitored weekly. Mice were fasted overnight $(12 \mathrm{~h})$ prior to measuring fasting blood glucose or performing glucose tolerance tests (GTT) and insulin tolerance tests (ITT). For GTT, glucose ( $1 \mathrm{~g} / \mathrm{kg}$ in saline) was administered by intraperitoneal (i.p.) injection. Blood glucose levels were measured before and 15, 30, 60, 120 min after glucose injection with a handheld Contour ${ }^{\text {TS }}$ glucometer (Bayer, Tarrytown, NY). For ITT, insulin $(0.75 \mathrm{U} / \mathrm{kg}$, Humulin R, Lilly, Indianapolis, IN) was administered by i.p. injection and blood glucose levels were measured before and 15, 30,60, 120 min after insulin injection.

\section{CTL Adoptive Transfer}

CTL adoptive transfer experiments were performed as described previously [35]. Briefly, CTLs were generated in vitro by culturing splenocytes and lymph node cells from OT-1 T cell receptor transgenic mice in IMDM $+10 \%$ FCS with $1 \mu \mathrm{M}$ OVA peptide (SIINFEKL) (provided by Dr. P. Allen, Washington University School of Medicine) for 5 days. Live cells were harvested using Ficoll-hypaque (GE Healthcare, Piscataway, NJ) and labeled with $1 \mu \mathrm{M}$ CFSE (Invitrogen) and injected intravenously $\left(10^{7}\right.$ cells/mouse). Epididymal adipose tissue and spleen were harvested $24 \mathrm{~h}$ after injection and processed for flow cytometry. 


\section{Statistical analyses}

Data were routinely presented as mean $+/$ - standard deviation (SD), and we determined significance by the Student's t test or ANOVA. We considered a $\mathrm{P}$ value of $<0.05$ as statistically significant.

\section{Results}

Induction of NKG2D ligands in fat tissue of obese mice

NKG2D binds to a number of distinct ligands, which are usually not expressed in normal adult tissues, but are up-regulated in response to various 'cellular stress conditions', such as oxidative stress, viral infection, and DNA damage [42]. The induced ligands are thought to act as a signal to the immune system for the removal of damaged cells. In order to identify factors that could potentially regulate NKG2D ligands in adipocytes, we incubated differentiated 3T3-L1 cells with factors that induce various cell responses. Insulin and TNF- $\alpha$ treatment caused a significant increase in the mRNA levels of different NKG2D ligands at varying concentrations. Insulin had the greatest effect on Rae-1 mRNA level among the NKG2D ligands (Fig. 1A) while TNF- $\alpha$ treatment increased both Rae-1 and Mult-1 (Fig. 1A and B). H60b mRNA levels were only moderately affected by insulin and TNF- $\alpha$ (Fig. 1C). Meanwhile, incubation in tunicamycin, glucose oxidase, LPS, and palmitate did not have an effect (Fig. 1D).

Insulin and TNF- $\alpha$ levels are known to be elevated during obesity in humans and rodents. To address whether NKG2D ligands are induced by obesity, we analyzed the adipose tissue of diet-induced obese (DIO) mice. RNA was isolated from epididymal adipose tissue obtained from mice fed either a normal diet (ND) or a high-fat diet (HFD) and analyzed by real-time qPCR. Rae-1 and Mult-1 mRNA levels were increased approximately 5fold and 17-fold respectively in the DIO-mice compared to lean control mice. (Fig. 2A). H60b expression level was not altered in DIO-mice. As control, we confirmed increased expression of CD68, TNF- $\alpha$, and MCP-1 and decreased expression of adiponectin and adipsin in DIO-mice (Fig. 2B).

To confirm these results, we also analyzed the adipose tissue of $o b / o b$ mice, a genetic model of obesity (Fig. 2C, D). Similar to the DIO-mice, Rae-1 and Mult-1 transcript levels were increased in the $o b / o b$ mice compared to lean $o b /+$ controls (Fig. 2C). In addition, H60b mRNA level was also slightly increased in the ob/ ob mice (Fig. 2C).

The adipose tissue is composed of adipocytes, immune cells, vascular cells, and undifferentiated preadipocytes. In order to determine which cell types express NKG2D ligands, the epidydymal adipose tissue of DIO-mice was fractionated into adipocytes and the stromal vascular fraction (SVF) via collagenase digestion. Rae-1 was expressed predominantly in the adipocyte fraction, while Mult-1 had similar expression levels in the adipocytes and the SVF (Fig. 2E). H60b expression was higher in the adipocyte fraction (Fig. 2E).

Induction of NKG2D ligands in the adipose tissue of obese mice led us to hypothesize that this could be a mechanism by which CD8 $\mathrm{T}$ cells are recruited to the adipose tissue to initiate inflammation during obesity. Infiltration of CD8 $\mathrm{T}$ cells to the adipose tissue of DIO-mice has been shown to occur relatively soon after starting the HFD regimen ( $4 \sim 5$ weeks after initiation of diet). In order to see whether NKG2D ligand induction occurs within a similar time frame, we measured the time-course kinetics of NKG2D ligand expression in DIO-mice (Fig. 2F). The mRNA levels of Rae-1 and Mult-1 were highest at the earliest time-point measured (3 weeks post-initiation of diet) and gradually decreased during the progression of obesity. These results suggest that early changes that occur in the adipose tissue of mice on a HFD induce the expression of NKG2D ligands.

Obesity-induced insulin resistance and adipose tissue inflammation in NKG2D-deficient mice. The NKG2Ddeficient $\left(k_{l r k} k^{--}\right)$mice lack the expression of NKG2D in all immune cells including CD8 T cells. To directly test whether NKG2D ligands are involved in the development of obesityinduced insulin resistance, we placed wild-type and $\mathrm{klrk}^{-/-}$mice on a HFD. Age-matched male mice were divided into two groups and placed on either ND or HFD. We measured the changes in body weight and fasting glucose levels for 18 weeks. In order to measure insulin sensitivity, we performed glucose tolerance test (GTT) and insulin tolerance test (ITT) at 6, 12, and 18 weeks after starting the diet regimen.

At baseline (8-weeks old), there was no difference between WT and $k_{l r k} k^{-/}$mice in body weight (Fig. 3A) and fasting blood glucose levels (Fig. 3B). Rapidly within a few weeks, mice on the HFD began to outweigh mice on the normal diet, and the gap between the two groups continued to grow until the end of the experiment. There was no difference in body weight change between WT and $k l r k^{-/}$mice in either the ND or HFD group throughout the experiment.

In GTT experiments (Fig. 3C), obese mice were significantly less tolerant compared to lean mice in both $\mathrm{WT}$ and $\mathrm{klrk}^{-/}$mice. However, there was no difference between WT and $k l r k^{-/}$mice in any of the time points. Similar results were obtained in ITT assays (Fig. 3D) with WT and $k l r k^{-/-}$mice displaying similar degrees of insulin sensitivity. Fasting glucose, which is also indicative of insulin sensitivity, was not altered in $k l r k^{-/}$mice. (Fig. 3B)

At the end of 18 weeks on the diet regimen, the epididymal adipose tissue was harvested to analyze immune cell infiltration. The SVF was isolated by collagenase digestion, stained for cell surface markers, and analyzed by flow cytometry. Consistent with previous reports, there was a significant increase in the number of $\mathrm{F} 4 / 80^{+} \mathrm{CD} 1 \mathrm{cc}^{+} \mathrm{M} 1$ macrophages and CD8 $\mathrm{T}$ cells in the adipose tissue of HFD-fed mice compared to lean counterparts (Fig. 4). However, the number of M1 macrophages and CD8 T cells in the adipose tissue were similar in WT and $k l r k^{-/}$mice. We also confirmed that there was no difference in CD8 $\mathrm{T}$ cell infiltration after 3 weeks of HFD, when NKG2D ligand expression was highest (data not shown). These results indicate that NKG2D does not affect CD8 $\mathrm{T}$ cell recruitment to the adipose tissue during obesity.

Obesity-induced adipose tissue inflammation in fatspecific Rae-1 $\varepsilon$ transgenic mice. In order to confirm these results, we generated a transgenic mice specifically over-expressing Rae-le in the adipose tissue (AdpnCre ${ }^{+} \mathrm{Rae}^{+}$) by crossing adiponectin-Cre mice [41] with PCGALL-Raele mice [35]. Real-time qPCR was used to confirm Rae-1 $\varepsilon$ over-expression in the adipose tissue (Fig. 5A). Rae-1 mRNA levels were not altered in liver and muscle (data not shown), indicating specific overexpression of Rae-1 $1 \varepsilon$ in the adipose tissue. It is known that NKG2D expression in NK cells and T cells is down-regulated in transgenic mice constitutively overexpressing NKG2D ligands $[43,44]$. Indeed, we observed loss of NKG2D expression in NK cells isolated from the adipose tissue, but not spleen or blood, again confirming fat-specific overexpression of Rae-1 in the Adpn ${ }^{+} \mathrm{Rae}^{+}$ mice (Fig. 5B).

To examine the early events of obesity-induced immune cell infiltration, these transgenic mice (AdpnCre ${ }^{+} \mathrm{Rae}^{+}$) were placed on a high-fat diet for 5 weeks, along with age-matched control mice lacking the transgene (AdpnCre ${ }^{+} \mathrm{Rae}^{-}$). At the end of 5 weeks, the SVF from the adipose tissue were analyzed by flow cytometry. Adipose tissue from obese mice contained approxi- 
A

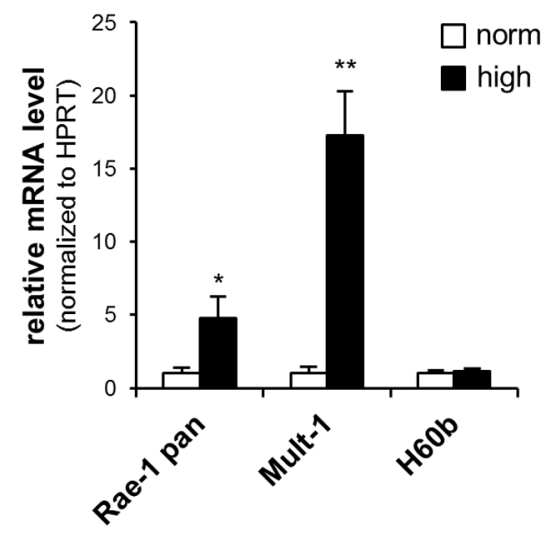

C

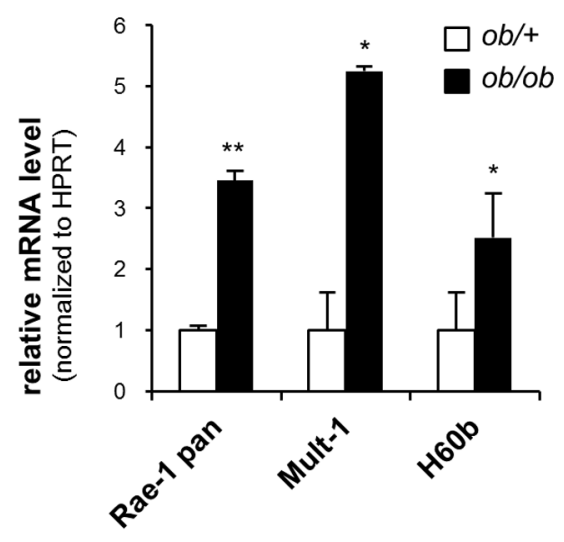

E

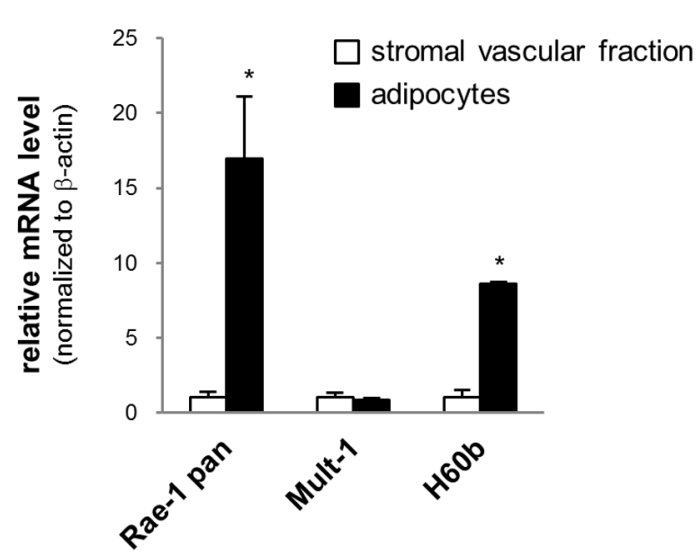

B

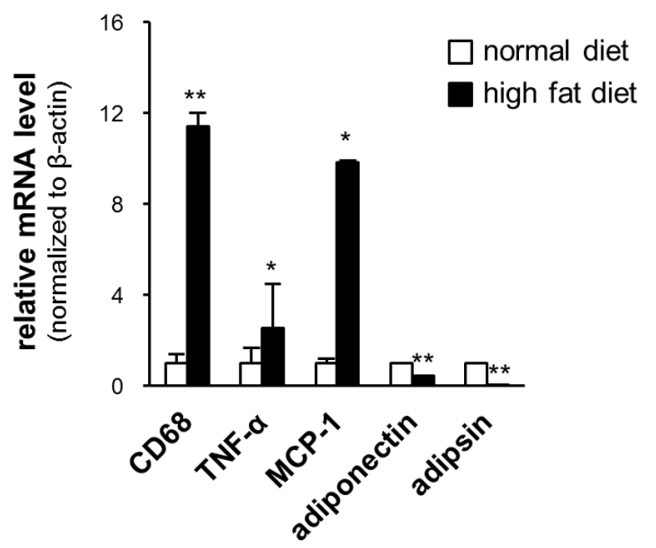

D

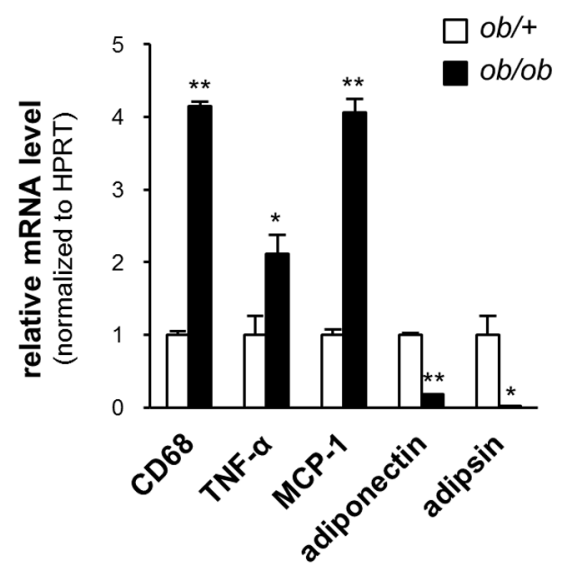

$\mathbf{F}$

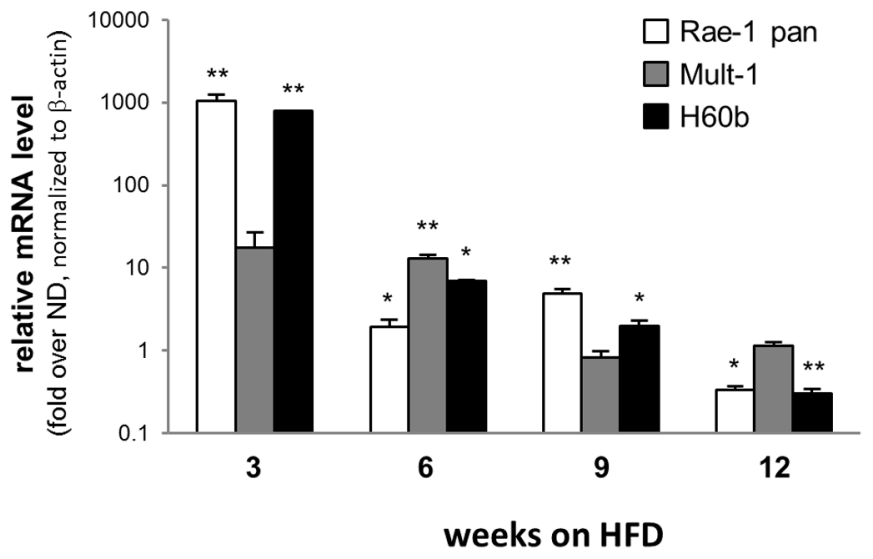

Figure 2. NKG2D ligands are increased in the adipose tissue of obese mice. Expression of NKG2D ligands in the epididymal adipose tissue from (A) DIO-mice (8 weeks on HFD) and (C) ob/ob mice was determined by quantitative PCR. mRNA levels of CD68, TNF- $\alpha$, MCP-1, adiponectin, and adipsin were measured in (B) DIO-mice and (D) ob/ob mice for control. (E) The epididymal adipose tissue of DIO-mice (6 weeks on HFD) was fractionated into the stromal vascular fraction and adipocytes and analyzed by quantitative PCR for expression of NKG2D ligands. (F) Adipocytes were obtained from the epipdidymal adipose tissue of DIO-mice at different time points after starting high-fat diet. The adipocytes were then analyzed by quantitative PCR for expression of NKG2D ligands. The results were normalized to (A-D) HPRT or (E, F) $\beta$-actin levels and are shown as fold-increase over $(A-D, F)$ lean control mice or (E) stromal vascular fraction. The data represent mean \pm SD of 3 independent experiments. ${ }^{*} p<0.05,{ }^{* *} p<0.01$ vs. $(A-D, F)$ lean control mice or $(E)$ stromal vascular fraction.

doi:10.1371/journal.pone.0110108.g002

mately twice the number of M1 macrophages and CD8 $\mathrm{T}$ cells compared to lean mice (Fig. 5C). However, the number of CD8 T cells and M1 macrophages in the adipose tissue was not altered by fat-specific overexpression of Rae- $1 \varepsilon$. These results are consistent with the observations made in $k l r k^{-/-}$mice and confirm that
NKG2D is not involved in the recruitment of CD8 T cells to the adipose tissue during obesity.

The $k l r k^{-/-}$mice and AdpnCre ${ }^{+} \mathrm{Rae}^{+}$mice used in the previous experiments were housed in specific pathogen-free conditions, and therefore, the number of circulating CTLs is minimal. In mice, NKG2D is expressed in activated CTLs, but not in naive CD8 T 
A

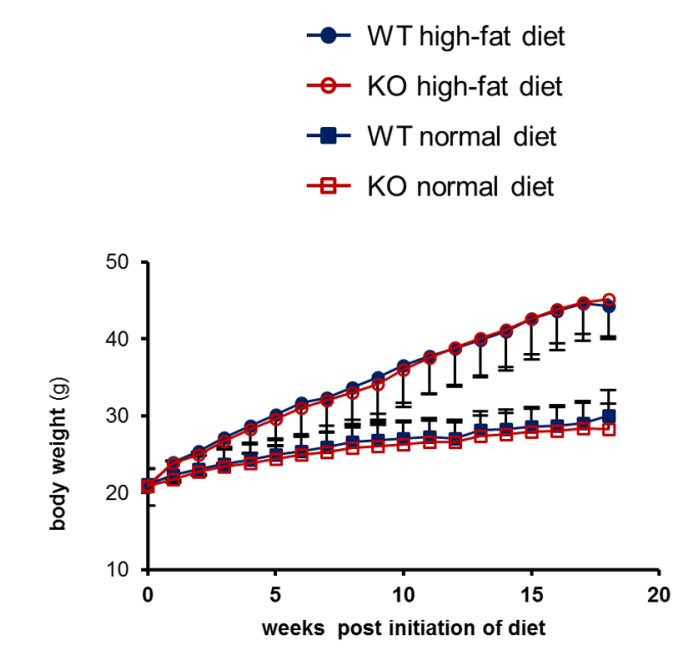

C

GTT
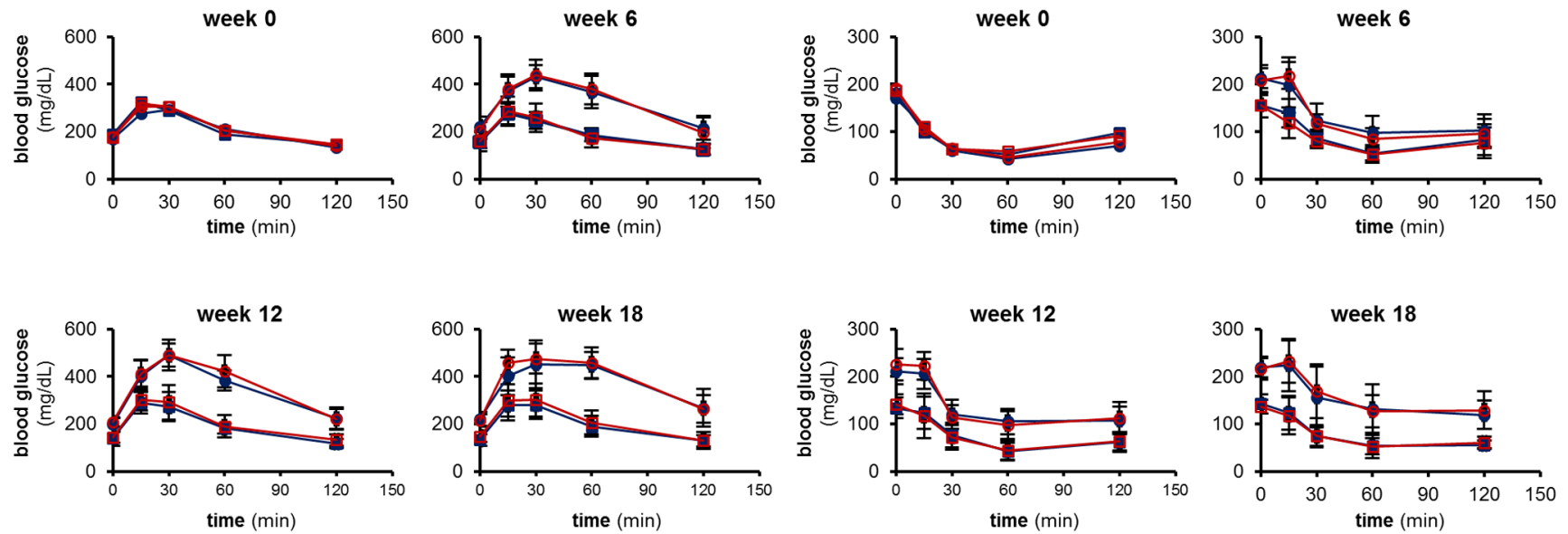

Figure 3. Susceptibility to obesity-induced insulin resistance is not altered in $\mathbf{k l r k}^{-1-}$ mice. (A) Body weight of WT and klrk ${ }^{-/-}$mice placed on normal diet or high-fat diet for 18 weeks. (B) Fasting blood glucose levels measured after 0,6 , 12, and 18 weeks on high-fat diet. (C) Glucose tolerance test and (D) insulin tolerance test performed after $0,6,12$, and 18 weeks on high-fat diet. The numbers of mice used for each experiments

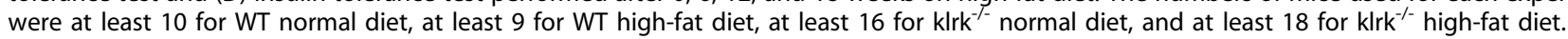
doi:10.1371/journal.pone.0110108.g003

cells. We therefore speculated that the lack of a phenotype could be due to low numbers of circulating CTLs. In addition, it was possible that the constitutive overexpression of Rae-1 $\varepsilon$ was downregulating NKG2D expression in circulating T cells. To circumvent these issues, and to directly test whether up-regulation of NKG2D ligands can induce recruitment and accumulation of cytotoxic CD8 $\mathrm{T}$ cells in the adipose tissue, we intravenously injected CFSE-labeled CTLs into control and AdpnCre ${ }^{+} \mathrm{Rae}^{+}$ mice. 24 hours after injection, we measured the number of CFSE ${ }^{+}$ CTLs recruited to the spleen and adipose tissue (Fig. 6). Similar numbers of $\mathrm{CFSE}^{+}$CTLs were detected in the spleen of AdpnCre ${ }^{+}$ $\mathrm{Rae}^{-}$and AdpnCre ${ }^{+} \mathrm{Rae}^{+}$mice, indicating that equal number of CTLs was successfully transferred to each mouse. In the adipose

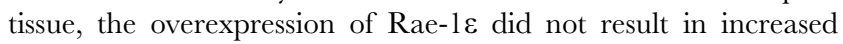
recruitment of CTLs. These results demonstrate that, in contrast to pancreatic islets, NKG2D-NKG2D ligand interaction is not sufficient to drive accumulation of NKG2D-expressing CTLs in the adipose tissue. 

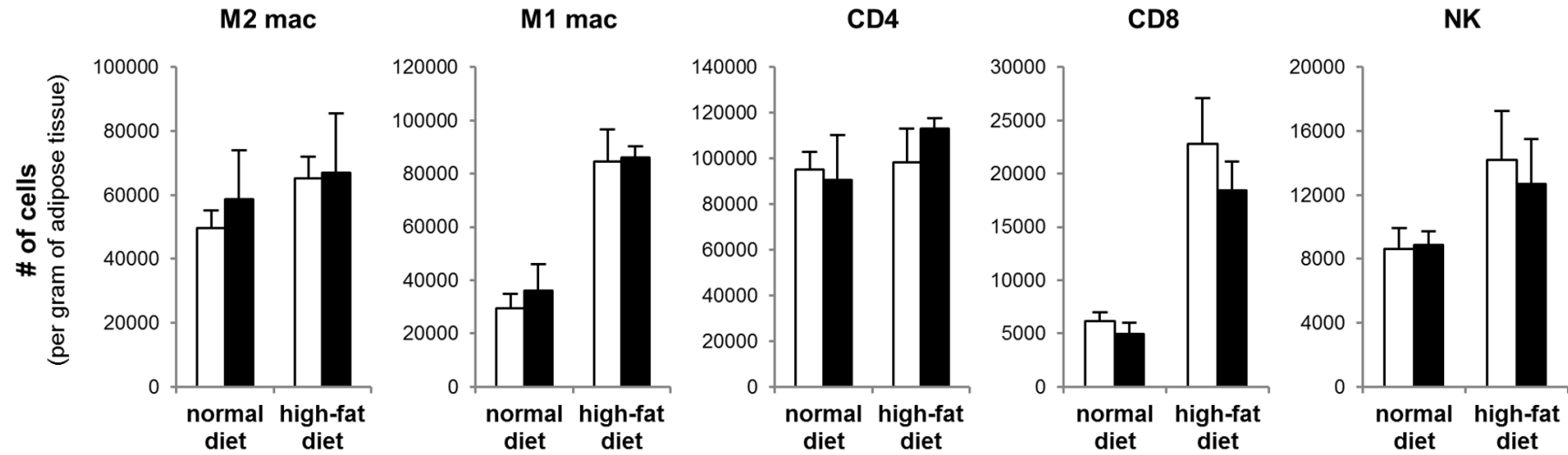

Figure 4. Immune cell infiltration of adipose tissue during obesity is not altered in $\mathbf{k l r k}^{-/-}$mice. The stromal vascular fraction was isolated from the epididymal adipose tissue of DIO and lean mice after 18 weeks on the diet regimen. The cells were stained with antibodies to identify M1 macrophages $\left(\mathrm{F} 4 / 80^{+}, \mathrm{CD} 11 \mathrm{c}^{+}\right), \mathrm{M} 2$ macrophages $\left(\mathrm{F} 4 / 80^{+}, \mathrm{CD} 11 \mathrm{c}^{-}\right), \mathrm{CD} 4 \mathrm{~T}$ cells $\left(\mathrm{CD} 3 \varepsilon^{+}, \mathrm{CD} 4^{+}\right), \mathrm{CD} 8 \mathrm{~T}$ cells $\left(\mathrm{CD} 3 \varepsilon^{+}, \mathrm{CD} 8^{+}\right)$, and NK cells $\left(\mathrm{CD} 3 \varepsilon^{-}, \mathrm{NK}_{1 .} 1^{+}\right)$ and analyzed by flow cytometry. The cells were gated on $7 A A D^{-}, C D 45^{+}$populations. The data represent mean \pm SD of 3 independent experiments. doi:10.1371/journal.pone.0110108.g004

\section{Discussion}

In this study, we tested a model of antigen-independent recruitment of $\mathrm{CD}^{+} \mathrm{T}$ cells to the adipose tissue during obesity via NKG2D-NKG2D ligand engagement. Results from our experiments suggest that interaction between the immunoreceptor NKG2D and its cognate ligands is not critically involved in the development of adipose tissue inflammation during diet-induced obesity. Neither deletion of NKG2D nor fat-specific overexpression of an NKG2D ligand had significant effects on insulin sensitivity or infiltration of immune cells to the adipose tissue in the setting of a high-fat diet.

The non-development of phenotype does not seem to be due to strain-specificity of the mice used in our experiments. The composition of immune cells is known to vary among different strains of mice [45]. In addition, the immune system is compromised in certain genetic obese mice models, such as the $o b / o b$ mice or the $d b / d b$ mice, because leptin is required for immune cell homeostasis, especially $\mathrm{T}$ cells [46]. In order to avoid such variables, we chose to utilize the diet-induced obese (DIO)mouse model using C57BL/6 mice. The C57BL/6 strain is the predominant strain used to study nutrient-dependent metabolic disorders in the literature and was the strain used to demonstrate the involvement of various immune cells in obesity-induced inflammation.

The NKG2D-deficient mice display a NK cell hyperproliferation phenotype [40], which could be a confounding factor in the case that NK cells are also involved in adipose tissue inflammation. However, as of date, NK cells have not been clearly implicated in obesity-induced insulin resistance. There have been conflicting reports of changes in the number of NK cells in the adipose tissue during obesity. In our experiments, we did not observe a clear reproducible pattern of NK cell recruitment.

Similar to the NKG2D-deficient mice, we did not observe any difference in immune cell infiltration in the fat-specific Rae-1 $\varepsilon$

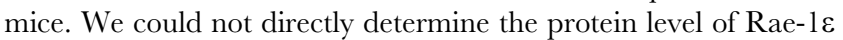
in the adipocytes of the fat-specific Rae-1 $\varepsilon$ mice due to the lack of antibodies suitable for immunohistochemistry or Western blots and the technical difficulties in performing flow cytometry with adipocytes. However, we did observe a nearly complete down- regulation of NKG2D specifically in NK cells isolated from the adipose tissue, but not in cells isolated from spleen or blood. It is well known that engagement of Raele by NKG2D results in NKG2D down-regulation [37,43,47]. Therefore, these results show that the level of transgene expression was sufficient to completely quench NKG2D expression on NK cells in the adipose tissue, suggesting that Rae- $1 \varepsilon$ is being expressed at biologicallyrelevant levels.

Although we observed induction of both Rae-1 and Mult-1 in obese mice, only Rae-1 was expressed predominantly in fat cells. It should be noted that adipocyte fractions isolated from adipose tissue of obese mice are often contaminated with lipid-laden immune cells, which could have affected the outcome of the NKG2D ligand expression analyses. Nonetheless, we speculated that overexpressing Rae-1 $\varepsilon$ in the adipose tissue would closely resemble the expression pattern of NKG2D ligands during obesity. It is possible that overexpressing Mult-1 instead could have yielded different results.

It has been shown that circulating levels of $\mathrm{CD}^{+} \mathrm{T}$ cells in male C57BL/6 mice are relatively low compared to other mouse strains. In addition, NKG2D is only expressed in activated CD8 ${ }^{+}$ $\mathrm{T}$ cells, the number of which may not be sufficient in mice raised in a SPF facility. In order to circumvent these issues, we adoptively transferred activated CTLs into the fat-specific Rae- $1 \varepsilon$ transgenic mice. Contrary to our expectations, overexpression of Rae- $1 \varepsilon$ in adipocytes did not lead to accumulation of CTLs in the adipose tissue. This is in contrast to previous results from similar experiments performed in the pancreatic islets [35]. This may be due to differences in the architecture of the vasculature between the pancreas and the adipose tissue, which could affect how surface proteins are detected by lymphocytes in circulation.

Despite the lack of evidence for the involvement of NKG2D in obesity-induced inflammation and insulin resistance, it is still interesting that NKG2D ligands are up-regulated in the adipose tissue in response to a high fat diet. In fact, the human NKG2D ligands MICA and MICB have been shown to be induced in the liver of obese patients [48]. In addition, NKG2D ligands are induced in the aorta of $A p o E^{-/-}$mice fed a western diet and are involved in atherosclerotic plaque formation [38]. The nature of 
A

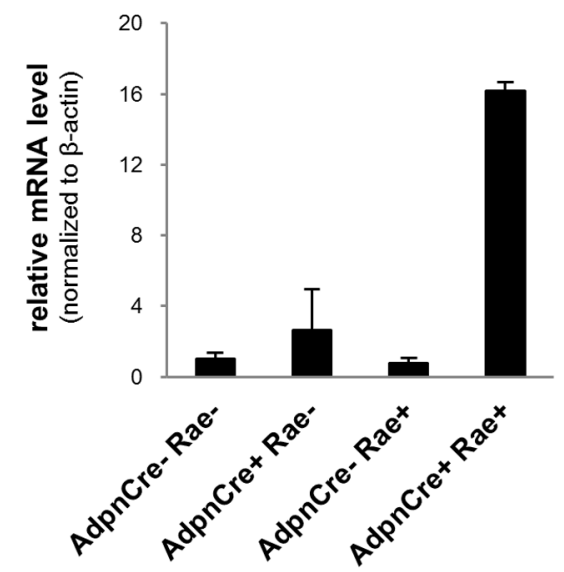

C

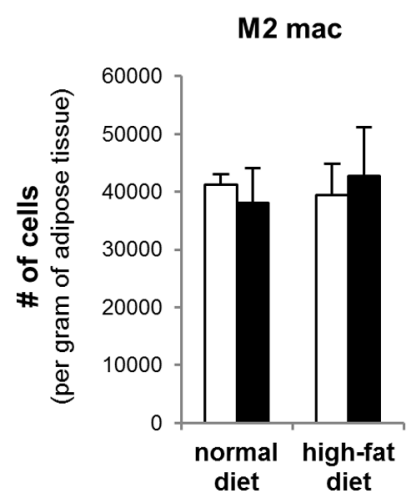

B
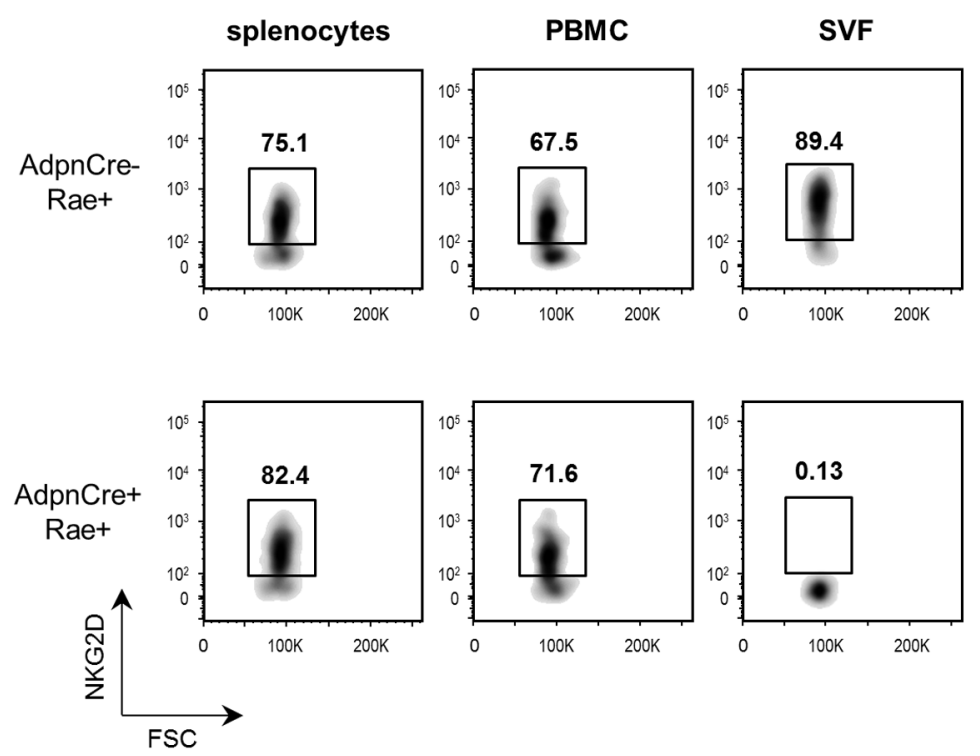

$\mathrm{AdpnCre}^{+} \mathrm{Rae}^{-}$

AdpnCre+ $\mathrm{Rae}^{+}$

CD4

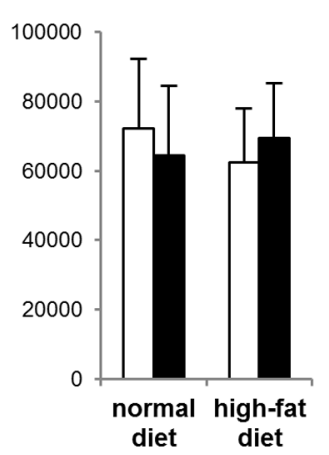

CD8

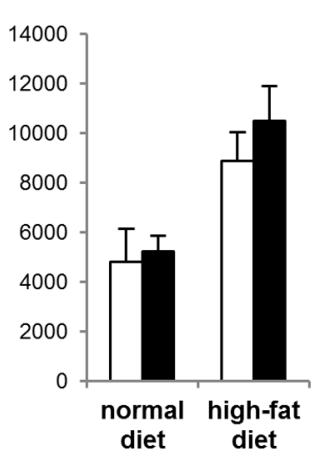

NK

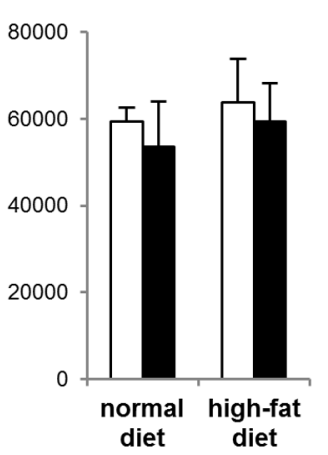

Figure 5. Immune cell infiltration of adipose tissue during obesity is not altered in AdpnCre Rae $^{+}$mice. (A) The epididymal adipose tissue from AdpnCre ${ }^{+} \mathrm{Rae}^{+}$and control mice were analyzed by quantitative PCR to determine Rae- 1 expression. The results were normalized to $\beta-$ actin levels and are shown as fold-increase over AdpnCre ${ }^{-}$Rae $^{-}$mice. (B) Splenocytes, peripheral blood mononuclear cells (PBMC), and stromal vascular fractions isolated from the epididymal adipose tissue of AdpnCre ${ }^{-} \mathrm{Rae}^{+}$and AdpnCre $\mathrm{Rae}^{+}$mice were stained with NKG2D antibodies and analyzed by flow cytometry. Cells were gated on 7AAD ${ }^{-}, \mathrm{CD}_{4} 5^{+}, \mathrm{CD} 3 \varepsilon^{-}, \mathrm{NK} 1.1^{+}$populations. (C) The stromal vascular fraction isolated from the epididymal adipose tissue of $\mathrm{AdpnCre}^{+} \mathrm{Rae}^{-}$and $\mathrm{AdpnCre}{ }^{+} \mathrm{Rae}^{+}$mice after 5 weeks on the diet regimen were stained with antibodies and analyzed by flow cytometry. The cells were gated on $7 \mathrm{AAD}^{-}, \mathrm{CD}^{2} 5^{+}$populations. The data represent mean $\pm \mathrm{SD}$ of 3 independent experiments. doi:10.1371/journal.pone.0110108.g005

the response of the immune system to NKG2D ligand induction necessitates a tight regulation of its expression. Previous studies have shown that various 'cell stress' conditions, including DNA damage and viral infection, can induce NKG2D ligand expression. However, it seems that such cues are not sufficient to up-regulate NKG2D ligands in all cell types. It is more likely that different signals trigger the expression of the multiple NKG2D ligands in different cell types. Indeed, insulin and TNF- $\alpha$ were able to significantly increase transcript levels of Rae-1 and Mult-1, but not H60b, in differentiated 3T3-L1 adipocytes. Notably, high-fat diet resulted in the up-regulation of the same subgroup of NKG2D ligands, namely Rae-1 and Mult-1. Therefore, it is tempting to speculate that these signals are also responsible for the induction of
NKG2D ligands in the adipose tissue in vivo given that insulin and TNF- $\alpha$ levels are known to be elevated in obesity.

Interestingly, multiple NKG2D ligands were expressed in undifferentiated 3T3-L1 cells and the expression levels decreased after differentiation (data not shown). This could be attributed to the cease of proliferation that occurs when 3T3-L1 cells differentiate. It was recently demonstrated that Rae-1 expression is regulated by the transcription factor $\mathrm{E} 2 \mathrm{~F}$, which is associated with cell cycle. On the other hand, it is also possible that NKG2D ligand expression is actively repressed by the differentiation process. Identification of such factors would shed light on how the strict regulation of NKG2D ligands is achieved in various cell types. 

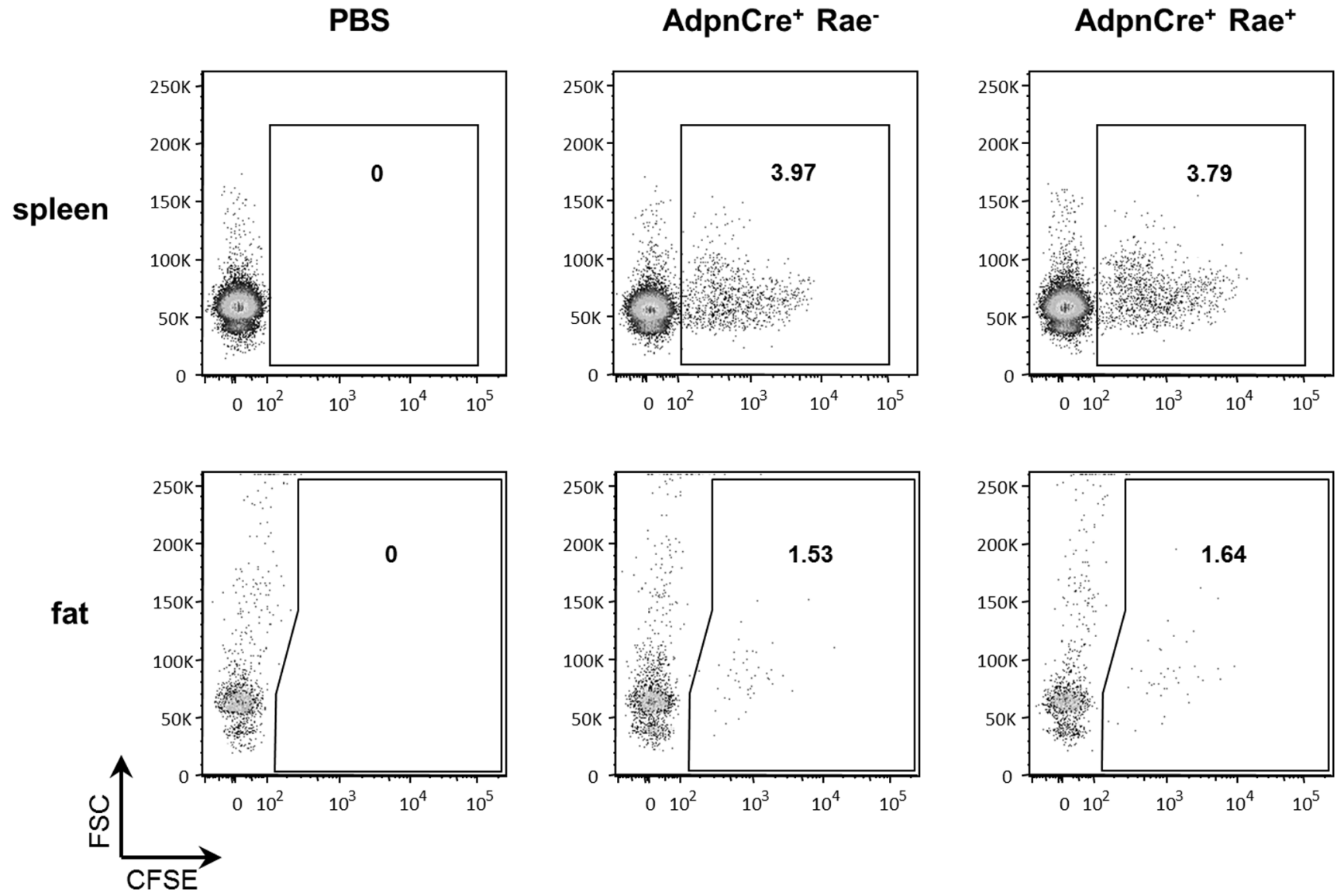

Figure 6. Rae-1 $\varepsilon$ overexpression does not induce CTL accumulation in the adipose tissue. CTLs generated from OT-1 mice were labeled with CFSE and injected intravenously. A group of mice were injected with PBS for control. Spleen and epididymal adipose tissue were isolated 24 hours after injection and analyzed by flow cytometry. Cells were gated on $7 A A D^{-}, C D 45^{+}, C D 3 \varepsilon^{+}$populations. The data are representative of at least 3 individual mice per group.

doi:10.1371/journal.pone.0110108.g006

The biological significance of NKG2D ligand induction in the adipose tissue during obesity is unclear. Adipocytes in the adipose tissue of HFD-fed mice are subjected to various cell stress conditions due to increased metabolic loads, hypoxic conditions, and proinflammatory environment. Hence, the most obvious explanation would be that NKG2D ligands are being expressed by damaged adipocytes. It should be noted that the different types of NKG2D ligands do not seem to respond in the same manner. Only Rae-1 and Mult-1 were induced by the introduction of the high-fat diet while H60b levels remained the same. Furthermore, Rae-1 was predominantly found in adipocytes whereas Mult-1 expression levels were similar in adipocytes and stromal vascular cells. How these differences affect adipose tissue homeostasis and/ or pathology during obesity remain to be determined.

In summary, our results rule out the interaction between NKG2D and its ligands as a possible mechanism of $\mathrm{CD}^{+} \mathrm{T}$ cell

\section{References}

1. Odegaard JI, Chawla A (2013) Pleiotropic actions of insulin resistance and inflammation in metabolic homeostasis. Science 339: 172-177.

2. Osborn O, Olefsky JM (2012) The cellular and signaling networks linking the immune system and metabolism in disease. Nat Med 18: 363-374.

3. Gregor MF, Hotamisligil GS (2011) Inflammatory mechanisms in obesity. Annu Rev Immunol 29: 415-445.

4. Hotamisligil GS, Shargill NS, Spiegelman BM (1993) Adipose expression of tumor necrosis factor-alpha: direct role in obesity-linked insulin resistance. Science 259: 87-91. recruitment to the adipose tissue during obesity. Our findings regarding the induction of NKG2D ligands in the adipose tissue during obesity provide a new model to investigate the mechanisms involved in the regulation of NKG2D ligand expression.

\section{Acknowledgments}

We would like to thank Dr. Trey Coleman (Washington University School of Medicine) for providing technical advice with adipose tissue fractionation.

\section{Author Contributions}

Conceived and designed the experiments: JC MM AS. Performed the experiments: JC. Analyzed the data: JC. Contributed reagents/materials/ analysis tools: BP. Wrote the paper: JC AS.

5. Hirosumi J, Tuncman G, Chang L, Gorgun CZ, Uysal KT, et al. (2002) A central role for JNK in obesity and insulin resistance. Nature 420: 333-336.

6. Cai D, Yuan M, Frantz DF, Melendez PA, Hansen L, et al. (2005) Local and systemic insulin resistance resulting from hepatic activation of IKK-beta and NF-kappaB. Nat Med 11: 183-190.

7. Yuan M, Konstantopoulos N, Lee J, Hansen L, Li ZW, et al. (2001) Reversal of obesity- and diet-induced insulin resistance with salicylates or targeted disruption of Ikkbeta. Science 293: 1673-1677. 
8. Arkan MC, Hevener AL, Greten FR, Maeda S, Li ZW, et al. (2005) IKK-beta links inflammation to obesity-induced insulin resistance. Nat Med 11: 191-198.

9. Barnes PJ, Karin M (1997) Nuclear factor-kappaB: a pivotal transcription factor in chronic inflammatory diseases. N Engl J Med 336: 1066-1071.

10. Karin M, Liu Z, Zandi E (1997) AP-1 function and regulation. Curr Opin Cell Biol 9: 240-246.

11. Ouchi N, Parker JL, Lugus JJ, Walsh K (2011) Adipokines in inflammation and metabolic disease. Nat Rev Immunol 11: 85-97.

12. Hosogai N, Fukuhara A, Oshima K, Miyata Y, Tanaka S, et al. (2007) Adipose tissue hypoxia in obesity and its impact on adipocytokine dysregulation. Diabetes 56: 901-911.

13. Ye J, Gao Z, Yin J, He Q (2007) Hypoxia is a potential risk factor for chronic inflammation and adiponectin reduction in adipose tissue of ob/ob and dietary obese mice. Am J Physiol Endocrinol Metab 293: E1118-1128.

14. Ozcan U, Cao Q, Yilmaz E, Lee AH, Iwakoshi NN, et al. (2004) Endoplasmic reticulum stress links obesity, insulin action, and type 2 diabetes. Science 306 : $457-461$.

15. Ozcan U, Yilmaz E, Ozcan L, Furuhashi M, Vaillancourt E, et al. (2006) Chemical chaperones reduce ER stress and restore glucose homeostasis in a mouse model of type 2 diabetes. Science 313: 1137-1140.

16. Yoshiuchi K, Kaneto H, Matsuoka TA, Kohno K, Iwawaki T, et al. (2008) Direct monitoring of in vivo ER stress during the development of insulin resistance with ER stress-activated indicator transgenic mice. Biochem Biophys Res Commun 366: 545-550.

17. Mathis D (2013) Immunological Goings-on in Visceral Adipose Tissue. Cell Metab 17: 851-859.

18. Olefsky JM, Glass CK (2010) Macrophages, inflammation, and insulin resistance. Annu Rev Physiol 72: 219-246.

19. Chawla A, Nguyen KD, Goh YP (2011) Macrophage-mediated inflammation in metabolic disease. Nat Rev Immunol 11: 738-749.

20. Weisberg SP, McCann D, Desai M, Rosenbaum M, Leibel RL, et al. (2003) Obesity is associated with macrophage accumulation in adipose tissue. $\mathrm{J}$ Clin Invest 112: 1796-1808.

21. Xu H, Barnes GT, Yang Q, Tan G, Yang D, et al. (2003) Chronic inflammation in fat plays a crucial role in the development of obesity-related insulin resistance. $\mathrm{J}$ Clin Invest 112: 1821-1830.

22. Lumeng CN, Bodzin JL, Saltiel AR (2007) Obesity induces a phenotypic switch in adipose tissue macrophage polarization. J Clin Invest 117: 175-184.

23. Martinez FO, Sica A, Mantovani A, Locati M (2008) Macrophage activation and polarization. Front Biosci 13: 453-461.

24. Wu H, Ghosh S, Perrard XD, Feng L, Garcia GE, et al. (2007) T-cell accumulation and regulated on activation, normal $\mathrm{T}$ cell expressed and secreted upregulation in adipose tissue in obesity. Circulation 115: 1029-1038.

25. Rausch ME, Weisberg S, Vardhana P, Tortoriello DV (2008) Obesity in C57BL/6J mice is characterized by adipose tissue hypoxia and cytotoxic T-cell infiltration. Int J Obes (Lond) 32: 451-463.

26. Kintscher U, Hartge M, Hess K, Foryst-Ludwig A, Clemenz M, et al. (2008) Tlymphocyte infiltration in visceral adipose tissue: a primary event in adipose tissue inflammation and the development of obesity-mediated insulin resistance. Arterioscler Thromb Vasc Biol 28: 1304-1310.

27. Rocha VZ, Folco EJ, Sukhova G, Shimizu K, Gotsman I, et al. (2008) Interferon-gamma, a Th1 cytokine, regulates fat inflammation: a role for adaptive immunity in obesity. Circ Res 103: 467-476.

28. Duffaut C, Galitzky J, Lafontan M, Bouloumie A (2009) Unexpected trafficking of immune cells within the adipose tissue during the onset of obesity. Biochem Biophys Res Commun 384: 482-485.
29. Caspar-Bauguil S, Cousin B, Galinier A, Segafredo C, Nibbelink M, et al. (2005) Adipose tissues as an ancestral immune organ: site-specific change in obesity. FEBS Lett 579: 3487-3492.

30. Nishimura S, Manabe I, Nagasaki M, Eto K, Yamashita H, et al. (2009) CD8+ effector $\mathrm{T}$ cells contribute to macrophage recruitment and adipose tissue inflammation in obesity. Nat Med 15: 914-920.

31. Winer S, Chan Y, Paltser G, Truong D, Tsui H, et al. (2009) Normalization of obesity-associated insulin resistance through immunotherapy. Nat Med 15: 921929.

32. Yang H, Youm YH, Vandanmagsar B, Ravussin A, Gimble JM, et al, (2010) Obesity increases the production of proinflammatory mediators from adipose tissue $\mathrm{T}$ cells and compromises TCR repertoire diversity: implications for systemic inflammation and insulin resistance. J Immunol 185: 1836-1845.

33. Feuerer M, Herrero L, Cipolletta D, Naaz A, Wong J, et al. (2009) Lean, but not obese, fat is enriched for a unique population of regulatory $\mathrm{T}$ cells that affect metabolic parameters. Nat Med 15: 930-939.

34. Cinti S, Mitchell G, Barbatelli G, Murano I, Ceresi E, et al. (2005) Adipocyte death defines macrophage localization and function in adipose tissue of obese mice and humans. J Lipid Res 46: 2347-2355.

35. Markiewicz MA, Wise EL, Buchwald ZS, Pinto AK, Zafirova B, et al. (2012) RAE1epsilon ligand expressed on pancreatic islets recruits NKG2D receptorexpressing cytotoxic $\mathrm{T}$ cells independent of $\mathrm{T}$ cell receptor recognition. Immunity 36: 132-141

36. Raulet DH (2003) Roles of the NKG2D immunoreceptor and its ligands. Nat Rev Immunol 3: 781-790.

37. Hotamisligil GS (2006) Inflammation and metabolic disorders. Nature 444: 860867.

38. Ye J (2009) Emerging role of adipose tissue hypoxia in obesity and insulin resistance. Int J Obes (Lond) 33: 54-66.

39. Song XM, Hresko RC, Mueckler M (2008) Identification of amino acid residues within the $\mathrm{C}$ terminus of the Glut4 glucose transporter that are essential for insulin-stimulated redistribution to the plasma membrane. J Biol Chem 283: 12571-12585.

40. Zafirova B, Mandaric S, Antulov R, Krmpotic A, Jonsson H, et al. (2009) Altered NK cell development and enhanced NK cell-mediated resistance to mouse cytomegalovirus in NKG2D-deficient mice. Immunity 31: 270-282.

41. Wang ZV, Deng Y, Wang QA, Sun K, Scherer PE (2010) Identification and characterization of a promoter cassette conferring adipocyte-specific gene expression. Endocrinology 151: 2933-2939.

42. Samarakoon A, Chu H, Malarkannan S (2009) Murine NKG2D ligands: "double, double toil and trouble". Mol Immunol 46: 1011-1019.

43. Oppenheim DE, Roberts SJ, Clarke SL, Filler R, Lewis JM, et al. (2005) Sustained localized expression of ligand for the activating NKG2D receptor impairs natural cytotoxicity in vivo and reduces tumor immunosurveillance. Nature immunology 6: 928-937.

44. Wiemann K, Mittrucker HW, Feger U, Welte SA, Yokoyama WM, et al. (2005) Systemic NKG2D down-regulation impairs NK and CD8 T cell responses in vivo. J Immunol 175: 720-729.

45. Petkova SB, Yuan R, Tsaih SW, Schott W, Roopenian DC, et al. (2008) Genetic influence on immune phenotype revealed strain-specific variations in peripheral blood lineages. Physiol Genomics 34: 304-314.

46. Lam QL, Lu L (2007) Role of leptin in immunity. Cell Mol Immunol 4: 1-13.

47. Ogasawara K, Hamerman JA, Hsin H, Chikuma S, Bour-Jordan H, et al. (2003) Impairment of NK cell function by NKG2D modulation in NOD mice. Immunity 18: 41-51.

48. Kahraman A, Schlattjan M, Kocabayoglu P, Yildiz-Meziletoglu S, Schlensak M, et al. (2010) Major histocompatibility complex class I-related chains A and B (MIC A/B): a novel role in nonalcoholic steatohepatitis. Hepatology 51: 92-102. 